\title{
Adaptive Filtering Applied to an Uplink Load Estimate in WCDMA
}

\author{
Erik Geijer Lundin, Fredrik Gunnarsson, Fredrik Gustafsson \\ Division of Communication Systems \\ Department of Electrical Engineering \\ Linköpings universitet, SE-581 83 Linköping, Sweden \\ WWW: http://www. control.isy.liu.se \\ E-mail: geijer@isy.liu.se, fred@isy.liu.se \\ fredrik@isy.liu.se
}

9th September 2003

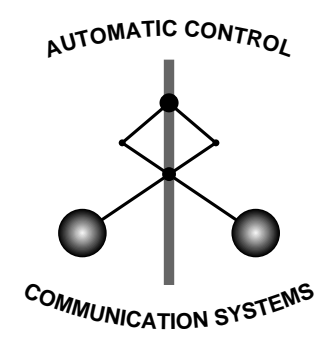

LINKÖPING

Report no.: LiTH-ISY-R-2545

Submitted to VTC'03 Spring

Technical reports from the Control \& Communication group in Linköping are available at http://www. control.isy.liu.se/publications. 


\begin{abstract}
In the uplink of a WCDMA system, a natural choice of resource quantity is the uplink noise rise, i.e., total received power over noise power. Unfortunately this quantity is hard to measure and estimates are often noisy. This paper focuses on relative load which is closely related to the noise rise. Model-based signal processing with change detection techniques is herein used to suppress noise and minor oscillations while being alert on fast load changes. The time varying model identified in the process can also be used for prediction of future values, something which resource management algorithms can benefit from.
\end{abstract}

Keywords: WCDMA, Adaptive Filtering, Uplink Load 


\title{
Adaptive Filtering Applied to an Uplink Load Estimate in WCDMA
}

\author{
Erik Geijer Lundin, Fredrik Gunnarsson and Fredrik Gustafsson* \\ Division of Communication Systems \\ Department of Electrical Engineering, Linköpings universitet \\ SE-581 83 LINKÖPING, SWEDEN \\ \{geijer, fred, fredrik\}@isy.liu.se
}

\begin{abstract}
In the uplink of a WCDMA system, a natural choice of resource quantity is the uplink noise rise, i.e., total received power over noise power. Unfortunately this quantity is hard to measure and estimates are often noisy. This paper focuses on relative load which is closely related to the noise rise. Model-based signal processing with change detection techniques is herein used to suppress noise and minor oscillations while being alert on fast load changes. The time varying model identified in the process can also be used for prediction of future values, something which resource management algorithms can benefit from.
\end{abstract}

\section{INTRODUCTION}

All cellular radio systems of today employ radio resource management algorithms to utilize the available resources as efficiently as possible. These algorithms depend on some sort of resource quantity. In the uplink of a WCDMA system a natural choice of such a quantity is the total received power, $I^{\text {tot }}$ [1]. $I^{\text {tot }}$ is related to uplink relative load, $L$, through the pole equation [2] (which will here be taken as a definition of L)

$$
I^{t o t} \triangleq \frac{N}{1-L}
$$

where $N$ is the background noise power. There are a number of different approaches to estimate the relative load. A wide spread approach is to consider the relative load as consisting of two parts, one part being the sum of contributions from users within the cell and the other part (originating from users outside the cell) being a fraction, $f$, of this internal load. This technique has been applied in [3], [4], [5]. Another, more straightforward technique is to use measurements of $I^{t o t}$, see e.g. [6].

Our approach has been to use a centralized estimate based on path gain measurements and an assumption of perfect power control, see below or [7], [8]. Regardless of the method used, the estimated uplink relative load will, just as the true uplink interference power, fluctuate over time. Figure 1 shows estimated and true uplink relative load. Clearly both of them experience oscillations. These oscillations are due to the constant user movement; as the users move around in the environment their number of soft handover links regularly changes. By using more soft handover links less interference power is induced into the system.

The above discussion motivates filtering of the estimated uplink load, both for suppressing noise due to measurement inaccuracies but also to provide a more stable signal. However, simply low pass filtering it could be very dangerous since we

${ }^{*}$ This work is supported by the Swedish Agency for Innovation Systems (VINNOVA), Information Systems for Industrial Control and Supervision (ISIS) and in cooperation with Ericsson Research, which are all acknowledged.

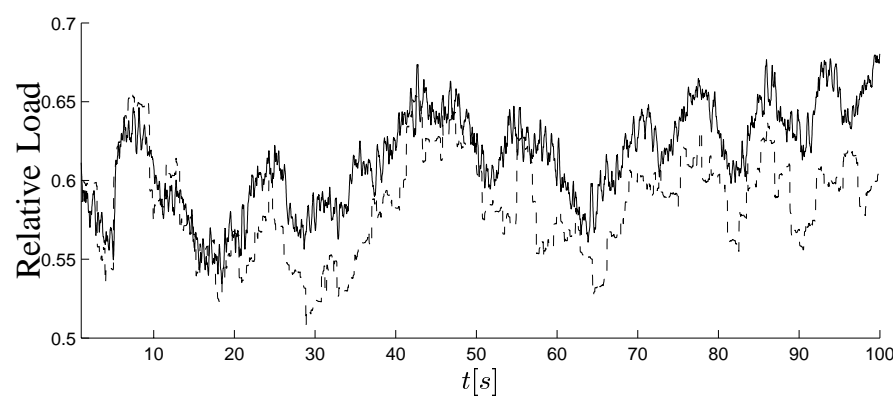

Fig. 1. Uplink relative load oscillates with a rather low frequency. Solid: filtered true relative load, dashed: estimated relative load.

need to be alert on sudden changes in the load. Therefore we have applied a signal model together with Kalman filtering and change detection to the relative load estimate in order to reduce noise and oscillations while keeping the sudden changes in the signal. The time varying model also provides an indication of towards where the load is currently heading, something which enables more aggressive resource management algorithms.

The rest of this paper is divided as follows. The next section contains a derivation of the uplink load estimates. Section III provides a presentation of the signal model used herein. In Section IV we present theory for Kalman filtering, change detection and discusse some of the filter design choices. Kalman filter and CUSUM detector are applied to simulated uplink load estimates in Section V. Finally some conclusions are made in Section VI.

\section{System ModeL}

This section will explain the system model assumed and then derive the estimates of the uplink relative load.

\section{A. Wireless Networks}

The uplink noise rise in base station $j$ (different cells are herein denoted as different base stations, even though they are connected to the same physical site), $\Lambda_{j}$, is defined as the total interference power in the base station, $I_{j}^{t o t}$, over background noise power, $N_{j}$. The total received interference power at time $t, I_{j}^{t o t}(t)$, is compiled by the background noise power and the sum of all the users' carrier powers

$$
I_{j}^{t o t}(t)=N_{j}(t)+\sum_{i=1}^{M} C_{i, j}(t)=\sum_{i=1}^{M} p_{i}(t) g_{i, j}(t)
$$

where $C_{i, j}(t)$ is the signal power from user $i$ received in base station $j, p_{i}(t)$ is user $i$ 's transmission power at time $t, g_{i, j}(t)$ is the path gain between user $i$ and base station $j$ and $M$ is 
the total number of users in the network. Assume that mobile $i$ is connected solely to base station $j$. The perceived quality is related to the carrier-to-interference-ratio (CIR) defined by $\gamma_{i}(t)=C_{i, j}(t) /\left(I_{j}^{t o t}(t)-C_{i, j}(t)\right)$. Power control in WCDMA aims at maintaining $\gamma_{i}(t)$ equal to a user dependent target $C I R, \gamma_{i}^{t g t}(t)$ [8]. These targets are continuously reconsidered by radio resource management algorithms. For presentational ease we will from here on use the carrier-to-total-interferenceratio (CTIR), $\beta_{i}(t)=C_{i, j}(t) / I_{j}^{t o t}(t)$ instead of $\gamma_{i}(t)^{1}$. In the system considered here, a mobile may be connected to several base stations at different physical sites (soft handover) or to several base stations at the same site (softer handover). Utilizing maximum ratio combining of the received signals in softer handover yields:

$$
\gamma_{i}(t)=\sum_{k \in K_{i}(t)} \frac{g_{i, k}(t) p_{i}(t)}{I_{k}^{t o t}(t)-g_{i, k}(t) p_{i}(t)}
$$

where $K_{i}(t)$ is the set of base stations user $i$ is connected to at time $t$. We, however, are interested in the combined $\beta_{i}(t)$ which is approximated by

$$
\beta_{i}(t) \approx \sum_{k \in K_{i}(t)} \frac{g_{i, k}(t) p_{i}(t)}{I_{k}^{t o t}(t)}
$$

Conversely, in the case of soft handover selection combining is used and consequently the signal with the best quality measure is chosen, i.e.

$$
\beta_{i}(t)=\max _{k \in K_{i}(t)} \frac{g_{i, k}(t) p_{i}(t)}{I_{k}^{t o t}(t)}
$$

Note that both soft and softer handover are used concurrently in a true system as well as in the simulator. Maximum in (4) will be taken over the results from the local softer handover combinings if only a subset of the active links are in softer handover. In the general case, where both soft and softer handover are used $\beta_{i}(t)$ may be expressed as

$$
\beta_{i}(t)=f\left(g_{i}(t), K_{i}(t), \boldsymbol{I}^{t o t}\right) p_{i}(t)
$$

where $f\left(g_{i}(t), K_{i}(t), \boldsymbol{I}^{\text {tot }}\right)$ combines user $i$ 's pathgains with the different $I_{k}^{t o t}$ in $\boldsymbol{I}^{\text {tot }}$ according to the combination of soft and softer handover defined by $K_{i}$. Thus, the function $f$ is a combination of equations (3) and (4), i.e., taking selection combining of the locally maximum ratio combined received signals.

\section{B. Uplink Load Estimate}

Solving for $p_{i}(t)$ in (5), assuming perfect power control $\left(\beta_{i}=\beta_{i}^{t g t}\right)$ and inserting it into (2) yield

$$
I_{j}^{t o t}=N_{j}+\sum_{i=1}^{M} g_{i, j} \frac{\beta_{i}^{t g t}}{f\left(g_{i}(t), K_{i}(t), \boldsymbol{I}^{t o t}\right)}
$$

Assuming the background noise the be equal in all base stations, i.e., $N_{j}=N_{k}=N$, and dividing equation (6) by $N$ provide the following system of equations, which in general is nonlinear (note that $f$ is homogeneous for positive numbers)

$$
\Lambda_{j}(t)=1+\sum_{i=1}^{M} \beta_{i}^{t g t}(t) \frac{g_{i, j}(t)}{f\left(g_{i}(t), K_{i}(t), \boldsymbol{\Lambda}(t)\right)}
$$

\footnotetext{
${ }^{1}$ Note the relation $\beta_{i}=\gamma_{i} /\left(1+\gamma_{i}\right)$.
}

One way of numerically solving this system of equations is to iterate; simply use the previous estimate in the right hand equation

$$
\hat{\Lambda}_{j}(t)=1+\sum_{i=1}^{M} \beta_{i}^{t g t}(t) \frac{g_{i, j}(t)}{f\left(g_{i}(t), K_{i}(t), \hat{\Lambda}(t-1)\right)}
$$

Along the way a couple of choices regarding pathgain combining and solution methods have been done, in [9] a number of similar estimates have been derived and evaluated. Using equation (1), the following holds

$$
\Lambda_{j} \triangleq \frac{I_{j}^{t o t}}{N_{j}}=\frac{1}{1-L_{j}} \Leftrightarrow L_{j}=1-\frac{1}{\Lambda_{j}}
$$

Thus an estimate of the uplink noise rise can easily be converted into an estimate of the uplink relative load.

\section{Signal Model}

When dealing with signals whose behaviour is expected to change, continuously or abruptly, a fixed model is obviously not attractive. Using a parameterized signal model and estimating not only the actual signal value but also the parameters of the model enables a more accurate signal estimate as well as an earlier detection of an abrupt change, in the parameters and/or the signal level. A signal $y(t)$, originating from an unbiased $n$ :th order Auto Regressive (AR)-model, may be described as (see e.g., [10])

$$
y(t)+a_{1} y(t-1)+a_{2} y(t-2)+\ldots+a_{n} y(t-n)=e(t)
$$

where $e$ is white Gaussian noise and $a_{1}, a_{2}, \ldots, a_{n}$ are the parameters of the model. $y(t)$ in equation (10) will have $E y(t)=0$, but the signals we study have $E y(t)=\bar{L}(t)$, therefore we need a slightly more complex model where the time-varying bias is subtracted from each $y(t)$ giving $E(y(t)-\bar{L}(t))=0$. Replacing $y(t)$ by $y(t)-\bar{L}(t)$ in $(10)$ yields

$$
(y(t)-\bar{L}(t))+\sum_{i=1}^{n} a_{i}(y(t-i)-\bar{L}(t-i))=e(t)
$$

[11] uses non-zero-mean Gaussian noise when deriving an expression for a biased AR model which is then used when modeling the amount of requested bandwidth in a wireless network. [12] presents another method of estimating the parameters of biased AR models.

We use a fourth order biased AR model to describe $y$, i.e., $n=4$ in (11). It is natural to assume different time scales of $L$ and $y$, otherwise the AR-model is ambigious. That is, the ARmodel takes care of short term oscialltions and $L(t)$ models the long term drifts. Then equation (11) may be rearranged to

$$
y(t)=\bar{L}(t)\left(1+\sum_{i=1}^{4} a_{i}\right)-\sum_{i=1}^{4} a_{i} y(t-i)+e(t)
$$


A state space representation of equation (12) in discrete time is

$$
\begin{gathered}
x(t)=\left[\bar{L}(t) \dot{\bar{L}}(t) a_{1} a_{2} a_{3} a_{4}\right]^{T} \\
x(t+1)=\left(\begin{array}{ccc}
1 & T & 0 \\
0 & 1 & 0 \\
0 & 0 & I_{4}
\end{array}\right) x(t)+\left(\begin{array}{cc}
\frac{T^{2}}{2} & 0 \\
T & 0 \\
0 & T I_{4}
\end{array}\right) w(t) \\
y(t)=\left(1+\sum a_{i} \quad 0 \quad-y(t-1) \cdots-y(t-4)\right) x+e(t)
\end{gathered}
$$

where $w(t)$ is a 5-dimensional vector containing the process noise. Note that the measurement $y(t)$ has a non-linear relation to the parameters. This makes the parameter estimation more difficult. By introducing two new variables, $\tilde{L}=\left(1+\sum a_{i}\right) \bar{L}$ and $\dot{\tilde{L}}$, we can convert the above nonlinear state space model to obtain a linear state space model

$$
\begin{gathered}
\tilde{x}(t)=\left[\tilde{L}(t) \dot{\tilde{L}}(t) a_{1} a_{2} a_{3} a_{4}\right]^{T} \\
\tilde{x}(t+1)=\left(\begin{array}{ccc}
1 & T & 0 \\
0 & 1 & 0 \\
0 & 0 & I_{4}
\end{array}\right) \tilde{x}(t)+\left(\begin{array}{cc}
\frac{T^{2}}{2} & 0 \\
T & 0 \\
0 & T I_{4}
\end{array}\right) w(t) \\
y(t)=\left(\begin{array}{ccc}
1 & 0 & -y(t-1) \cdots-y(t-4)) \tilde{x}(t)+e(t)
\end{array}\right.
\end{gathered}
$$

Denote by $Q$ the covariance matrix for the vector $w(t)$. Q will then be a diagonal matrix with elements $q_{1}, q_{2} \cdots q_{5}$ in the diagonal. The values $q_{2}$ to $q_{5}$ are all the same and much less than $q_{1}$. Hence we encourage changes in $\tilde{L}$ and $\dot{\tilde{L}}$ rather than in the AR-parameters. The innovations, $e(t)$ are assumed to be zero mean Gaussian with constant variance $R$.

As we are interested in $\bar{L}$ the following conversions are necessary to obtain the correct final result,

$$
\begin{gathered}
\hat{\bar{L}}(t)=\frac{\overline{\tilde{L}}}{1+\sum_{i=1}^{4} \tilde{a}_{i}}=\frac{\hat{\tilde{x}}_{1}}{1+\sum_{k=3}^{6} \hat{\tilde{x}}_{k}}=f(\hat{\tilde{x}}) \\
\operatorname{Var} \hat{\bar{L}}=f_{\hat{\tilde{x}}}(\hat{\tilde{x}})^{T} \operatorname{Cov} \hat{\tilde{x}} f_{\hat{\tilde{x}}}(\hat{\tilde{x}})
\end{gathered}
$$

\section{AdAPTIVE FILTERING}

In this section we will explain the techniques used in the filtering. The behaviour of both the Kalman filter and the change detection algorithm are defined by a number of parameters. We will also discuss the design choices made when setting the values of these parameters.

\section{A. Kalman Filtering}

Equation (13) is of the form

$$
\begin{gathered}
x(t+1)=A x(t)+B_{v} w(t) \\
y(t)=C(t) x(t)+e(t)
\end{gathered}
$$

We want to estimate the state vector $x(t)$ in the above state space model such that the covariance of the state error, $E(\hat{x}(t)-x(t))(\hat{x}(t)-x(t))^{T}$, is minimized given measurements $y(t)$. Introduce the notation $\hat{x}(t \mid \tau)$ for the estimate of $x(t)$ given measurements up until time $\tau$. The notation is justified by the fact that at each time instant there is a measurement update, where the new measurement is considered, and a time update, where the predictions are updated. A natural way of updating the state estimate with a new measurement is

$$
\hat{x}(t \mid t)=\hat{x}(t \mid t-1)+K(t) \epsilon(t)
$$

where $\epsilon(t)=y(t)-C(t) \hat{x}(t \mid t-1)$ is called the residual at time $t$. Out of all possible ways of choosing $K(t)$, the Kalman filter [13] chooses $K(t)$ such that the covariance of the residual, $\epsilon(t)$, is minimized. This optimality holds if the noise vectors are Gaussian, otherwise the Kalman filter is the best possible linear filter. $K(t)$ is chosen based on knowledge of the measurement errors' variance, $R=E e(t) e(t)^{T}$, the process noise covariance, $Q=E w(t) w(t)^{T}$, and an estimate of the covariance of the current estimation error, $P(t \mid \tau)=$ $E(x-\hat{x}(t \mid \tau))(x-\hat{x}(t \mid \tau))^{T}$. Since $P$ is time variant it, too, has to be updated continuously. The update of $P, K$ and $\hat{x}$ is done according to

$$
\begin{array}{rll}
\hline \text { Algorithm } 1 \text { (Kalman filter) } & \\
\cline { 1 - 1 } & & \\
\hat{x}(t \mid t) & =\hat{x}(t \mid t-1) C(t)^{T}\left(C(t) P(t \mid t-1) C(t)^{T}+R\right)^{-1} \\
P(t \mid t) & =P(t \mid t-1)-K(t) C(t) P(t \mid t-1) & (15 \mathrm{a}) \\
\hat{x}(t+1 \mid t) & =A \hat{x}(t \mid t) & (15 \mathrm{c}) \\
P(t+1 \mid t) & =A P(t \mid t) A^{T}+B_{v} Q B_{v}^{T} & (15 \mathrm{~d}) \\
\hat{x}(0 \mid-1) & =\hat{x}_{0}, P(0 \mid-1)=P_{0}
\end{array}
$$

Equations (15a) and (15b) are the measurement update, whereas (15c) and (15d) are the time update. $Q$ as well as $R$ are design parameters of the filter. We are interested in filtering $y(t)$, so we study $x(t \mid t)$.

Consequently, the Kalman filter delivers an estimate of the state vector $x(t)$, an estimated covariance matrix for this estimate (indicating the accuracy of the estimate), $P(t)$, and a residual $\epsilon(t)$ at each time instant $t$.

\section{B. Change Detection}

An always present problem with traditional estimation is the trade off between tracking and noise suppression. A way of getting around this problem is by studying the residuals, $\epsilon(t)$, of a process estimating $\tilde{x}$. In the model we use, the residuals are expected to be zero mean Gaussian, i.e., $e(t) \in N(0, R)$. If the estimates of $e(t), \epsilon(t)$, are not zero-mean Gaussian we have reason to believe there has been an abrupt change in one or several of the parameters.

In order to detect a bias in the residuals, the squared normalized residuals (normalized with their estimated standard deviation), $\bar{\epsilon}^{2}$, are fed to a distance measurement algorithm. At every time instant this algorithm adds the normalized residual minus a drift term $\nu$ to the previous accumulated distance. Hence, several consecutive residuals with considerable large magnitude will result in a growing distance $g(t)$, and eventually a change detection once $g$ has exceeded a fix threshold, $h$. The cumulative sum (CUSUM) algorithm is explained by the following pseudo code which is run through each time step

\begin{tabular}{l}
\hline Algorithm 2 (CUSUM) \\
\hline$g=g+\bar{\epsilon}^{2}-\nu$ \\
if $g<0$
\end{tabular}




$$
\begin{aligned}
& \qquad g=0 \\
& \text { end } \\
& \text { if } g>h \\
& g=0 \\
& \text { flag for detected change } \\
& \text { end }
\end{aligned}
$$

Once a change is detected, the filter characteristics is somehow temporarily changed in order to quickly adapt to the new situation. For more information on adaptive filtering and change detection see [14].

\section{Design Choices}

Figure 2 shows an example of a raw signal produced by the load estimation (i.e., equations (8) and (1)). The period of the slowest oscillations is about 20 seconds. The sampling time of this signal is $T_{\text {frame }}=0.01$ seconds, thus the slowest oscillations have a period of about 2000 samples. To describe the correlation in a signal having oscillations with such a long period would require thousands of parameters. Another way of looking at it is that when using far too few AR parameters, the difference between the samples considered by the AR model is simply noise. A result of using too short time horizon is an estimated model which only integrates noise. To put it mathematically, the model will have a pole close to +1 . A pole close to one means that $q=1$ satisfies the characteristic equation of the AR model

$$
1+a_{1} q^{-1}+a_{2} q^{-2} \cdots a_{n} q^{-n}=0
$$

Thus, if the sum of the estimated AR parameters is close to minus one, it is likely that the time horizon is too short.

A rule of thumb is that the period should be approximately 10 samples [10]. Therefore we use every 200:th sample when constructing the regression vector, $C(t)$. This is a kind of down sampling which requires an anti alias filter to be applied. The chosen low pass filter is a second order filter with both its poles on the real axis.

$$
y(t)=\frac{4 \cdot 10^{-4}}{1-1.96 q^{-1}+0.96 q^{-2}} L(t)
$$

This results in a filter which does not introduce any additional oscillations but on the other hand does not have a linear phase shift. Since we are interested in the low frequencies of the signal, a linear phase shift is not a requirement here. The choice of filterbandwidth is a trade off between low alias effects and low time delay between input and output of the antialias filter.

The state space representation in (13) has to be slightly modified to adapt to the resampling. Thus the final state space model used in the simulations is

$$
\begin{aligned}
& \tilde{x}(t)=\left[\begin{array}{llllll}
\tilde{L}(t) & \dot{\tilde{L}}(t) & a_{1} & a_{2} & a_{3} & a_{4}
\end{array}\right]^{T} \\
& \tilde{x}(t+1)=\left(\begin{array}{ccc}
1 & T & 0 \\
0 & 1 & 0 \\
0 & 0 & I_{4}
\end{array}\right) \tilde{x}(t)+\left(\begin{array}{cc}
\frac{T^{2}}{2} & 0 \\
\stackrel{T}{T} & 0 \\
0 & T I_{4}
\end{array}\right) w \\
& y(t)=\left(\begin{array}{lllll}
1 & 0 & -y(t-200) & \cdots & -y(t-800)
\end{array}\right) \tilde{x}(t)+e(t)
\end{aligned}
$$

Note that $y(t)$ is the output from the anti alias filter, and $T$ is the update rate of the filter times the length of a frame,

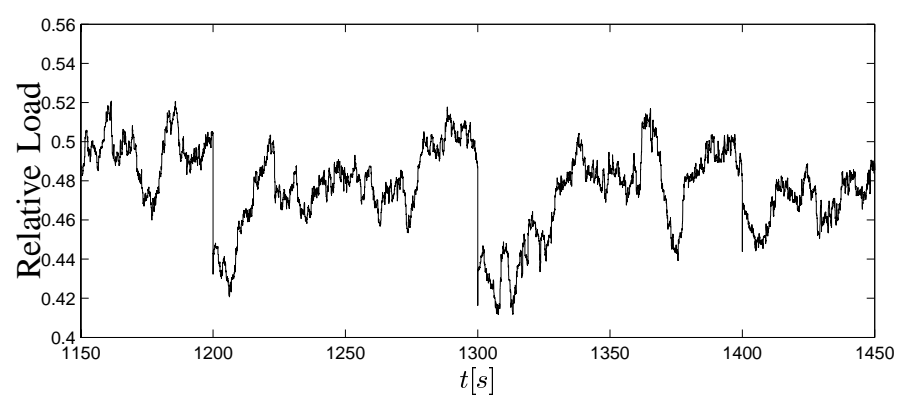

Fig. 2. Example of $L(t)$.

$T_{\text {frame }}=0.01 \mathrm{~s}$. The Kalman filter is parameterized by the constants in the matrix $Q$ and the scalar $R$, which thus can be seen as filter design variables. A larger value in one of $Q$ 's components means that the corresponding state variable(s) are more willing to change during the time between two time instants, whereas a larger $R$ corresponds to less measurement accuracy. Hence, larger $Q$ results in larger $K$ and larger $R$ results in a filter which puts less trust in the measurements and $K$ is chosen smaller yielding a "slower" filter. The choice of the size of $Q$ versus the size of $R$ is thus a trade off between tracking and noise suppression. $R$ should be chosen such that it approximately equals the variance of the residuals.

Another design choice concerns the update rate of the filter. A reason for not choosing a faster update rate, which of course provides a possibility for earlier detection of large changes in the relative load, is the increased computational burden a higher update rate induces. So perhaps updating every sample is not an obvious choice. We have studied filter that has an update rate of every 10:th or every 100:th estimate sample. Simulations show that the performance in steady state when filtering is the same regardless of the update rate.

The behaviour of the CUSUM detector is primarily decided by the parameters $h$ and $\nu$. These have been chosen in such a way that we detect sudden changes of considerable amplitude within a reasonable time without having too many false alarms. The choice of $h$ is dependent on the update rate of the filter. We have chosen to scale $h$ with the update rate.

$$
h=\frac{T}{T_{\text {frame }}} h_{0}
$$

where $h_{0}$ is the threshold used when updating every sample. In case of a change detection, the values of $P$ corresponding to $\tilde{x}_{1}$ and $\tilde{x}_{2}$ are increased a factor five, which results in making filtered value of these states more sensitive to the current input to the filter, $y(t)$. The motivation for increasing only these two states is that the change is believed to be in the load level and not in the AR parameters.

\section{Simulations}

The estimate used as input to this paper is from a simulation scenario consisting of 21 cells where multiple services are provided. We have used a Kalman filter to extract the load level $\bar{L}$ from the signal $y$ in equation (12). By using the change detection technique described in section IV we can easily follow any sudden jumps in the load estimate.

The top plot of figure 3 shows the raw estimate, i.e., $L$ in (1) where $\Lambda$ has been estimated using (8), and the output of 
the Kalman filter. The filter output is updated every frame, so $T=T_{\text {frame }}=0.01 \mathrm{~s}$. The filtered estimate is free of oscillations and follows an underlying trend in the original estimate. Notice, how the filtered estimate jumps when there is a distinct jump in the raw estimate. The middle plot of figure 3 shows a close up version of the top plot together with a rough low pass filtered version of the original estimate. Both filters suppress noise equally well, but notice the low pass filtered signal's slow adaptation to the new load level. In case of a sudden jump upwards, the slow acclimatization of the low pass filtered signal can be very dangerous. In case of a jump downwards, we can improve the resource utilization by admitting more load earlier if we use the output from the Kalman filter as input to a resource management algorithm compared to using the low pass filtered version. As a comparison, the bottom plot of figure 3 shows the performance of the filter when using three different update rates; once every frame as above, once every 10:th frame and once every hundred frame. In steady state, the three versions of the filtering provides almost identical outputs. But, due to its superior update rate, the version which is updated every frame detects the jump earlier. However, when updating every 100:th frame detects the jump even before the every 10:th frame update version in this particular instant. Thus, a faster update rate is perhaps not crucial, especially when comparing with the low pass filtered version which is also shown in the same plot. Considering the increased computational burden a faster update rate introduces, the faster update rate is probably not worth its price. The estimation process also provides us
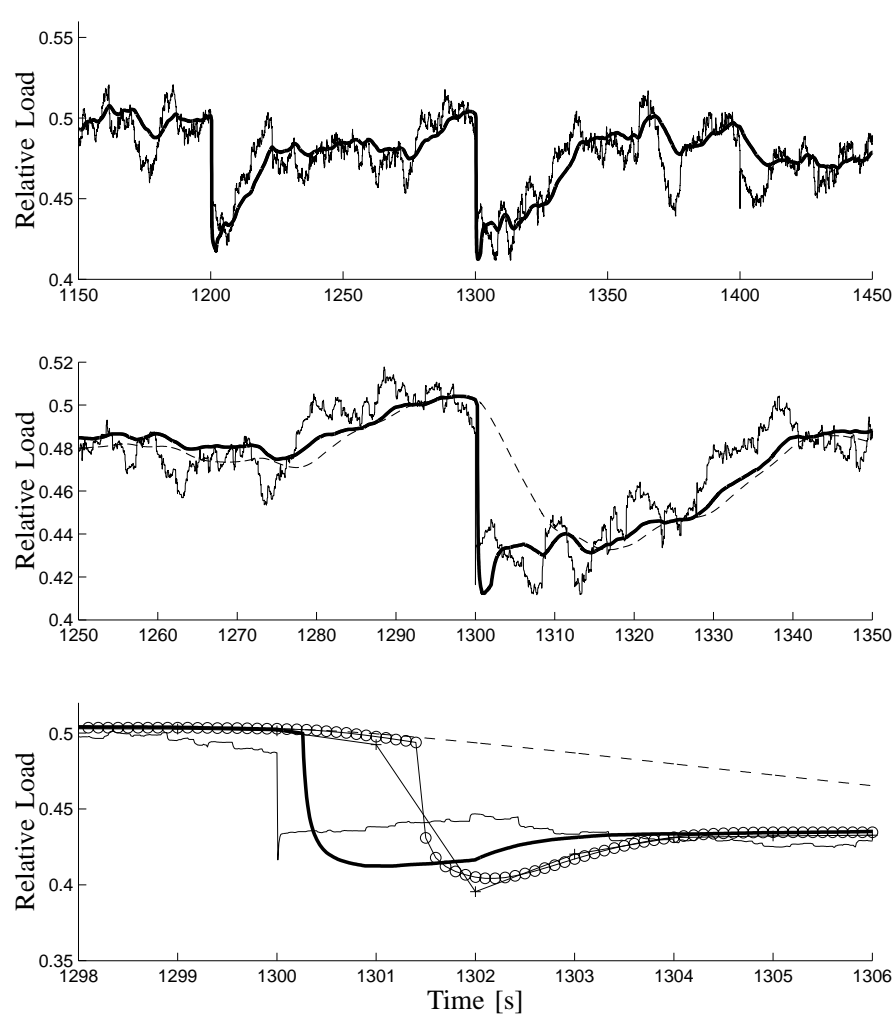

Fig. 3. Solid:original estimate. Solid, thick: updated each frame. Solid, ring: updated every 10:th frame. Solid, plus: updated every 100:th frame. Dashed: low pass filtered

with an estimate of the derivative of the trend in the relative load which enables a more aggressive resource management. Due to the anti alias filter applied before the Kalman filter, the measurement noise $e(t)$ is no longer white. Therefore the residuals from the estimation process are not white either. This indeed indicates the need for a more complex model, for example an ARMA model. However, as the simulations shows, we still manage to provide a good estimate of the underlying trend in the relative load estimate.

\section{CONCLUSIONS}

Due to user movement, the uplink relative load is subject to slow oscillations. Earlier work has provided an estimate of the uplink relative load which to a great extent captures these oscillations. In this paper we have proposed a signal model, which describes the relative load as a signal consisting of a bias with a trend together with a fourth order auto regressive model. A Kalman filter together with a change detection algorithm has been applied to the model. The result is a stable signal representing a time average of the uplink load. Unlike what an ordinary low pass filter would provide, we are still alert on sudden changes in the load level due to the change detection algorithm used. Furthermore, the filter provides us with an indication towards where the relative load is heading which enables a more aggressive resource management.

\section{REFERENCES}

[1] C. Y. Huang and R. D. Yates, "Call admission in power controlled CDMA systems," in Proc. IEEE Vehicular Technology Conference, Atlanta, GA, USA, May 1996.

[2] H. Holma and J. Laakso, "Uplink admission control and soft capacity with MUD in CDMA," in Proc. IEEE Vehicular Technology Conference, Amsterdam, the Netherlands, Sep 1999.

[3] A. Abrardo, G. Benelli, G. Giambene, and D. Sennati, "Performance analysis of a packet scheduling policy for a DS-CDMA cellular system," in Proc. IEEE Vehicular Technology Conference, Rhodes, Greece, May 2001.

[4] J. Sanchez, J. Perez-Romero, O. Sallent, and R. Agusti, "Mixing conversational and interactive traffic in the UMTS radio access network," in Proc.IEEE 4th International Workshop on Mobile and Wireless Communications Network, 2002, Sep 2002, pp. 597-601.

[5] V. Veeravalli, A. Sendonaris, and N. Jain, "CDMA converage, capacity and pole capacity," in Proc. IEEE Vehicular Technology Conference, Phoenix, AZ, USA, May 1997.

[6] W. Ying, Z. Jingmei, W. Weidong, and Z. Ping, "Call admission control in hierarchi cell structure," in Proc. IEEE Vehicular Technology Conference, Birmingham, AL, USA, may 2002.

[7] F. Gunnarsson, E. Geijer Lundin, G. Bark, N. Wiberg, "Uplink admission control in WCDMA based on relative load estimates," in Proc. IEEE International Conference on Communications, New York, USA, April 2002.

[8] F. Gunnarsson, "Power control in cellular radio system: Analysis, design and estimation," Ph.D. dissertation, Linköpings universitet, Linköping, Sweden, April 2000.

[9] E. Geijer Lundin, F. Gunnarsson, F. Gustafsson, "Uplink load estimation in WCDMA," in Proc. IEEE Wireless Communications and Networking Conference, New Orleans, USA, March 2003.

[10] L. Ljung, System Identification - Theory for the User, 2nd ed. Englewood Cliffs, NJ, USA.: Prentice Hall, 1999.

[11] M. Alasti and N. Farvardin, "A diffusion approximation for voice-data integration in a wireless network," in Proc. IEEE Wireless Communications and Networking Conference, Chicago, IL, USA, SEP 2000.

[12] C. Ngo and J. Mendel, "A higher-order moment formula for nonzero-mean AR processes," in Proc. IEEE International Conference on Acoustics, Speech, and Signal Processing, Toronto, Ont., Canada, Apr 1991.

[13] R. Kalman, "A new approach to linear filtering and prediction problems," Journal of Basic Engineering, Trans. of the ASME, vol. 82D:35-45, Mar 1960.

[14] F. Gustafsson, Adaptive filtering and change detection. John Wiley \& Sons, Ltd, 2000. 